\title{
Microscopic Analysis of Raman Images of Pharmaceutical Products Using Point Mapping and Line Scanning Method.
}

\author{
Eunah Lee*, Fran Adar*, and Andrew Whitley* \\ *Horiba Jobin Yvon, 3880 Park Ave., Edison, NJ 08820, USA
}

Raman imaging takes advantage of the excellent sensitivity and specificity of the Raman technique, especially when high spatial resolution is available by employing a confocal microscope. One largely overlooked aspect of point mapping technology is its flexibility. The area, shape and the spacing between spatial data points are all customizable to fit the characteristics of the sample and the goal of the project without losing the spectral resolution or signal intensity. A pharmaceutical over-the-counter analgesic tablet was selected and measured to demonstrate the impacts of the optimized experimental conditions on the image, spectra and information content.

The LabRam ARAMIS (Horiba Jobin Yvon, Edison, NJ, USA) was used to measure all samples. The excitation light was $632.8 \mathrm{~nm}$ line of the He/Ne laser. The confocal hole was opened to a diameter of $300 \propto \mathrm{m}$ and the slit to a width of $150 \propto \mathrm{cm}$. The $1200 \mathrm{gr} / \mathrm{mm}$ grating was used to measure the spectral range of $263.5-1151.2 \mathrm{~cm}^{-1}$. All data were measured using LabSpec 5 (Horiba Jobin Yvon, Edison, NJ, USA), and processed with LabSpec5 and ISys 4.0 (Spectral Dimensions, Inc., Olney, MD, USA).

The target sample (a commercial tablet of over-the-counter analgesic) contains three ingredients of $10 \%, \sim 40 \%$ and $\sim 50 \%$, respectively. Based on the size and weight of the sample, the chemical domains (areas where a single chemical component concentration is significantly higher than surrounding areas as to be identifiable as a 'domain' of the specified component) were estimated to be of the order of $100 \propto \mathrm{m}$ in diameter.

To identify the region of interest where spatial heterogeneity is significant, a coarse map of 200 . $200 \mathrm{~cm}^{2}$ at $25 \propto \mathrm{m}$ increments between measurement points was obtained. The area measured in this map is being considered as the total sampling area and the locations of subsequently measured maps are subsets of this map. At each spatial point, a spectrum was obtained by measuring and averaging two accumulations of $1 \mathrm{~s}$ exposure signal. The total data collection time for this measurement was approximately $4 \mathrm{~min}$. Raman chemical images were created from spectral lines of each species. The representative spectra of each species are shown in Figure 1c. It is noteworthy that, while each spectrum is unique, it is difficult to find a 'clean' band that does not overlap with spectral features of other species. Multivariate analysis results will be employed to overcome this obstacle.

For each species, a set of cursors was selected between which integrated intensities were calculated for each spatial point spectrum [271.5 - 315.0 (red), 767.3 - 802.8 (green), and 1034.0 - 1060.9 (blue)]. By subtracting a linear baseline defined by the cursors, the color-coded intensity correlated to the selected species. The composite chemical image is shown in Figure 1a. The rectangular insets indicate two areas that show particularly high spatial heterogeneity. These areas were measured again with the increasing increments $(2.5,1$ and $0.5 \propto \mathrm{cm})$ for further study. The data were processed and visualized the same way and will be reported. 
Raman chemical images of various sizes and increments from different locations as well as a fine grid map of the entire sampling area (2.5 $\mathrm{cm}$ increments and $8 \mathrm{hr}$ data collection, Figure $1 \mathrm{~b})$ demonstrate the relationship between information content and experimental configurations. We will further explore decreasing the measurement times of Raman images with small spatial increments $(<$ $1 \propto \mathrm{cm}$ ) while maintaining high spectral and spatial resolution by employing 'correct' hardware (e.g. rapid line scanning method and Piezo stage)) and data processing protocol.
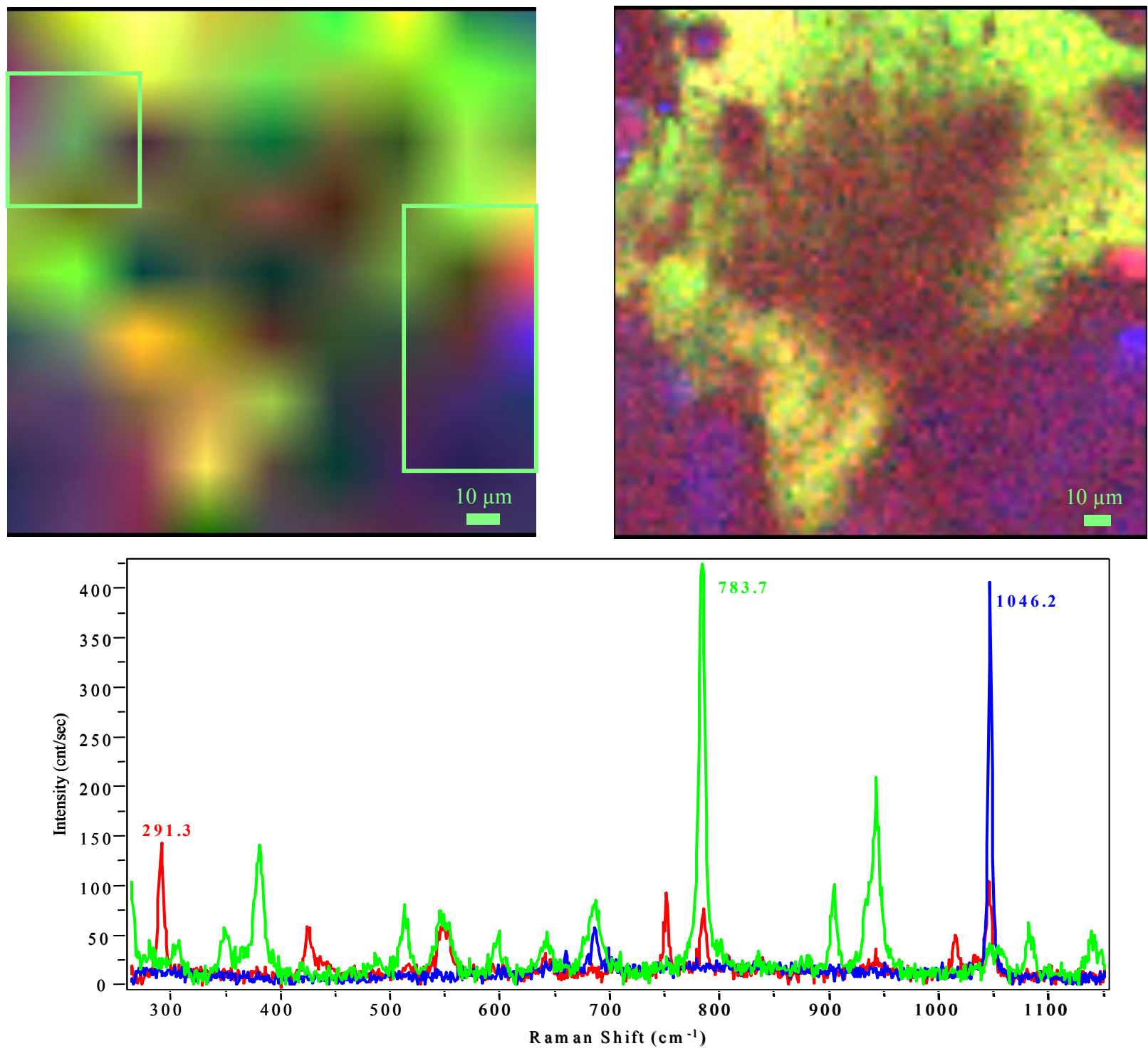

Figure 1. The composite chemical image is created from three Raman images of $200 \cdot 200 \propto \mathrm{cm}^{2}$ area with (a) 25 and (b) $2.5 \mathrm{~cm}$ increments. Red image is the integrated intensity map over the range of $271.5-315.0 \mathrm{~cm}^{-1}$. Linear baseline connecting two end points was subtracted. Green and blue images are similarly created for the regions of $767.3-802.8$ and $1034.0-1060.9 \mathrm{~cm}^{-1}$. (c) Point spectra representative for each chemical species. Red spectrum is from a red spot, green spectrum from a green spot, and blue spectrum from a blue spot. 